\title{
GAIA Level 4 Prenatally Diagnosed Congenital Microcephaly
}

National Cancer Institute

\section{Source}

National Cancer Institute. GAIA Level 4 Prenatally Diagnosed Congenital Microcephaly.

NCI Thesaurus. Code C128766.

GAIA Level 4 Prenatally Diagnosed Congenital Microcephaly is defined by three criteria: first, the fetus is at least 24 weeks gestational age (GA), with GA based on certain last menstrual period (LMP) date with confirmatory first or second trimester ultrasound (US) scan, OR uncertain LMP with second trimester ultrasound, intrauterine insemination (IUI), OR embryo transfer, OR certain or uncertain LMP with fundal height and no confirmatory first or second trimester scan; second, HC 2 SD below mean or less than 3 percentile according to fetal US scan using appropriate standardized reference charts according to GA and gender for the population (e.g., WHO growth reference charts if GA greater than or equal to 37 weeks and Intergrowth-21st reference charts for GA 24-36 weeks); third, $\mathrm{HC}$ at birth or autopsy is in the normal range using appropriate standardized reference charts according to GA and gender for the population, which means that this is NOT a case of prenatally diagnosed congenital microcephaly. 